\title{
An improved Kalai-Kleitman bound for the diameter of a polyhedron
}

\author{
Michael J. Todd * \\ September 3, 2018
}

\begin{abstract}
Kalai and Kleitman [6] established the bound $n^{\log (d)+2}$ for the diameter of a $d$-dimensional polyhedron with $n$ facets. Here we improve the bound slightly to $(n-d)^{\log (d)}$.
\end{abstract}

\section{Introduction}

A $d$-polyhedron $P$ is a $d$-dimensional set in $\mathbb{R}^{d}$ that is the intersection of a finite number of half-spaces of the form $H:=\left\{x \in \mathbb{R}^{d}: a^{T} x \leq \beta\right\}$. If $P$ can be written as the intersection of $n$ half-spaces $H_{i}, i=1, \ldots, n$, but not fewer, we say it has $n$ facets and these facets are the faces $F_{i}=P \cap H_{i}, i=1, \ldots, n$, each affinely isomorphic to a $(d-1)$-polyhedron with at most $n-1$ facets. We then call $P$ a $(d, n)$-polyhedron.

We say $v \in P$ is a vertex of $P$ if there is a half-space $H$ with $P \cap H=\{v\}$. (A polyhedron is pointed if it has a vertex, or equivalently, if it contains no line.) Two vertices $v$ and $w$ of $P$ are adjacent (and the set $[v, w]:=\{(1-\lambda) v+\lambda w: 0 \leq \lambda \leq 1\}$ an edge of $P$ ) if there is a half-space $H$ with $P \cap H=[v, w]$. A path of length $k$ from vertex $v$ to vertex $w$ in $P$ is a sequence $v=v_{0}, v_{1}, \ldots, v_{k}=w$ of vertices with $v_{i-1}$ and $v_{i}$ adjacent for $i=1, \ldots, k$. The distance from $v$ to $w$ is the length of the shortest such path and is denoted $\rho_{P}(v, w)$, and the diameter of $P$ is the largest such distance,

$$
\delta(P):=\max \left\{\rho_{P}(v, w): v \text { and } w \text { vertices of } P\right\} .
$$

We define

$$
\Delta(d, n):=\max \{\delta(P): P \text { a }(d, n) \text {-polyhedron }\}
$$

and seek an upper bound on $\Delta(d, n)$. It is not hard to see that $\Delta(d, \cdot)$ is monotonically nondecreasing. Also, the maximum above can be attained by a simple polyhedron, one where each vertex lies in exactly $d$ facets. See, e.g., Klee and Kleinschmidt [12] or Ziegler [18]. A related paper, Ziegler [19], gives the history of the Hirsch conjecture that $\Delta_{b}(d, n) \leq n-d$, where $\Delta_{b}(d, n)$ is defined as above but for bounded polyhedra.

In the last few years, there has been an explosion of papers related to the diameters of polyhedra and related set systems. Santos [14 found a counterexample to the Hirsch

*School of Operations Research and Information Engineering, Cornell University, Ithaca, NY 14853, USA. E-mail mjt7@cornell.edu. 
conjecture, later refined by Matschke, Santos, and Weibel [13. Eisenbrand, Hähnle, Razborov, and Rothkoss [3] showed that a slightly improved Kalai-Kleitman bound, $n^{\log (d)+1}$, held for a very general class of set families abstracting properties of the vertices of $d$-polyhedra with $n$ facets, which included the ultraconnected set families considered earlier by Kalai [4]. (This improved bound, for polyhedra, was presented first in Kalai [5].) Another class of set families was introduced by Kim [7; adding various properties gave families for which this bound held, or other families where the maximum diameter grew exponentially. The latter result is due to Santos [15]. Earlier combinatorial abstractions of polytopes include the abstract polytopes of Adler and Dantzig [1] (these satisfy the Hirsch conjecture for $n-d \leq 5$ ) and the duoids of [16, 17] (these have a lower bound on their diameter growing quadratically with $n-d$ ). We also mention the nice overview articles of Kim and Santos [8] (pre-counterexample) and De Loera [2] (post-counterexample).

Our bound $(n-d)^{\log (d)}$ fits better with the Hirsch conjecture and is tight for dimensions 1 and 2. Also, more importantly, it is invariant under linear programming duality. A pointed $d$-polyhedron with $n$ facets can be written as $\left\{x \in \mathbb{R}^{d}: A x \leq b\right\}$ for some $n \times d$ matrix $A$ of full rank and some $n$-vector $b$. Choosing an objective function $c^{T} x$ for $c \in \mathbb{R}^{d}$ gives the linear programming problem $\max \left\{c^{T} x: A x \leq b\right\}$, whose dual is $\min \left\{b^{T} y: A^{T} y=c, y \geq 0\right\}$. The feasible region for the latter is affinely isomorphic to a pointed polyhedron of dimension at most $n-d$ with at most $n$ facets, and equality is possible. Hence duality switches the dimensions $d$ and $n-d$.

\section{Result}

We prove

Theorem 1 For $1 \leq d \leq n, \Delta(d, n) \leq(n-d)^{\log (d)}$, with $\Delta(1,1)=0$.

(All logarithms are to base 2 ; note that $(n-d)^{\log (d)}=d^{\log (n-d)}$ as both have logarithm $\log (d) \cdot \log (n-d)$. We use this in the proof below.)

The key lemma is due to Kalai and Kleitman [6], and was used by them to prove the bound $n^{\log (d)+2}$. We give the proof for completeness.

Lemma 1 For $2 \leq d \leq\lfloor n / 2\rfloor$, where $\lfloor n / 2\rfloor$ is the largest integer at most $n / 2$,

$$
\Delta(d, n) \leq \Delta(d-1, n-1)+2 \Delta(d,\lfloor n / 2\rfloor)+2 .
$$

Proof: Let $P$ be a simple $(d, n)$-polyhedron and $v$ and $w$ two vertices of $P$ with $\delta_{P}(v, w)=\Delta(d, n)$. We show there is a path in $P$ from $v$ to $w$ of length at most the right-hand side above. If $v$ and $w$ both lie on the same facet, say $F$, of $P$, then since $F$ is affinely isomorphic to a $(d-1, m)$-polyhedron with $m \leq n-1$, we have $\rho_{P}(v, w) \leq \rho_{F}(v, w) \leq \Delta(d-1, m) \leq \Delta(d-1, n-1)$ and we are done.

Otherwise, let $k_{v}$ be the largest $k$ so that there is a set $\mathcal{F}_{v}$ of at most $\lfloor n / 2\rfloor$ facets with all paths of length $k$ from $v$ meeting only facets in $\mathcal{F}_{v}$. This exists since all paths of length 0 meet only $d$ facets (those containing $v$ ), whereas paths of length $\delta(P)$ can meet all $n$ facets of $P$. Define $k_{w}$ and $\mathcal{F}_{w}$ similarly. We claim that $k_{v} \leq \Delta(d,\lfloor n / 2\rfloor)$ and similarly for $k_{w}$. Indeed, let $P_{v} \supseteq P$ be the $\left(d, m_{v}\right)$-polyhedron $\left(m_{v}=\left|\mathcal{F}_{v}\right| \leq\lfloor n / 2\rfloor\right)$ defined by just those linear inequalities corresponding to the facets in $\mathcal{F}_{v}$. Consider any vertex $t$ of $P$ a distance $k_{v}$ from $v$, so there is a shortest path from $v$ to $t$ of length $k_{v}$ meeting only 
facets in $\mathcal{F}_{v}$. But this is also a shortest path in $P_{v}$, since if there were a shorter path, it could not be a path in $P$, and thus must meet a facet not in $\mathcal{F}_{v}$, a contradiction. So

$$
k_{v}=\delta_{P_{v}}(v, t) \leq \Delta\left(d, m_{v}\right) \leq \Delta(d,\lfloor n / 2\rfloor) .
$$

Now consider the set $\mathcal{G}_{v}$ of facets that can be reached in at most $k_{v}+1$ steps from $v$, and similarly $\mathcal{G}_{w}$. Since both these sets contain more than $\lfloor n / 2\rfloor$ facets, there must be a facet, say $G$, in both of them. Thus there are vertices $t$ and $u$ in $G$ and paths of length at most $k_{v}+1$ from $v$ to $t$ and of length at most $k_{w}+1$ from $w$ to $u$. Then

$$
\begin{aligned}
\Delta(d, n) & =\rho_{P}(v, w) \\
& \leq \rho_{P}(v, t)+\rho_{G}(t, u)+\rho_{P}(w, u) \\
& \leq k_{v}+1+\Delta(d-1, n-1)+k_{w}+1 \\
& \leq \Delta(d-1, n-1)+2 \Delta(d,\lfloor n / 2\rfloor)+2
\end{aligned}
$$

since, as above, $G$ is affinely isomorphic to a $(d-1, m)$-polyhedron with $m \leq n-1$.

Proof of the theorem: This is by induction on $d+n$. The result is trivial for $n=d$, since there can be only one vertex. Next, the right-hand side gives 1 for $d=1$ $(n=2)$ and $n-2$ for $d=2$, which are the correct values. For $d=3$, it gives $(n-3)^{\log (3)}$, which is greater than the correct value $n-3$ established by Klee [9, 10, 11]. (We could make the proof more self-contained by establishing the $d=3$ case from the lemma: a general argument deals with $n \geq 13$, but then there are seven more special cases to check.) Below we will give a general inductive step for the case $d \geq 4, n-d \geq 8$. Also, the result clearly holds by induction if $n<2 d$, since then any two vertices lie on a common facet, so their distance is at most $\Delta(d-1, n-1)$. The remaining cases are $d=4,8 \leq n \leq 11 ; d=5,10 \leq n \leq 12 ; d=6,12 \leq n \leq 13$; and $d=7, n=14$. All these cases can be checked easily using the lemma, the equation $\Delta(d, d)=0$, and the equations $\Delta(5,6)=\Delta(4,5)=\Delta(3,4)=\Delta(2,3)=1$.

Now we deal with the case $d \geq 4, n-d \geq 8$. For this, $\log (n-d) \geq 3$, so we have

$$
\begin{aligned}
\Delta(d, n) & \leq \Delta(d-1, n-1)+2 \cdot \Delta(d,\lfloor n / 2\rfloor)+2 \\
& \leq(d-1)^{\log (n-d)}+2 \cdot d^{\log (n / 2-d)}+2 \\
& \leq\left(\frac{d-1}{d}\right)^{\log (n-d)} d^{\log (n-d)}+2 \cdot d^{\log ((n-d) / 2)}+2 \\
& \leq\left(\frac{d-1}{d}\right)^{3} d^{\log (n-d)}+\frac{2}{d} \cdot d^{\log (n-d)}+2 \\
& =\left(1-\frac{3}{d}+\frac{3}{d^{2}}-\frac{1}{d^{3}}+\frac{2}{d}\right) d^{\log (n-d)}+2 \\
& \leq\left(1-\frac{1}{d}+\frac{3}{4 d}-\frac{1}{d^{3}}\right) d^{\log (n-d)}+2 \\
& \leq d^{\log (n-d)}-\frac{1}{4 d} \cdot d^{\log (n-d)}-\frac{1}{d^{3}} \cdot d^{\log (n-d)}+2 \\
\leq & d^{\log (n-d)},
\end{aligned}
$$

since each of the subtracted terms is at least one. This completes the proof.

Acknowledgement Thanks to Günter Ziegler and Francisco Santos for several helpful comments on a previous draft. 


\section{References}

[1] I. Adler and G.B. Dantzig, Maximum diameter of abstract polytopes, Mathematical Programming Study 1 (1974), 20-40.

[2] J.A. De Loera, New insights into the complexity and geometry of linear optimization, Optima, the newsletter of the Mathematical Optimization Society 87 (2011), 1-13.

[3] F. Eisenbrand, N. Hähnle, A. Razborov, and T. Rothvoss, Diameter of polyhedra: limits of abstraction, Mathematics of Operations Research 35 (2010), 786-794.

[4] G. Kalai, Upper bounds for the diameter and height of graphs of convex polyhedra, Discrete and Computational Geometry 8 (1992), 363-372.

[5] G. Kalai, Linear programming, the simplex method, and simple polytopes, Mathematical Programming 79 (1997), 217-233.

[6] G. Kalai and D.J. Kleitman, A quasi-polynomial bound for the diameter of graphs of polyhedra, Bulletin of the American Mathematical Society 26 (1992), 315-316.

[7] E.D. Kim, Polyhedral graph abstractions and an approach to the linear Hirsch conjecture, Mathematical Programming 143 (2013), 357-370.

[8] E.D. Kim and F. Santos, An update on the Hirsch conjecture, Jahresbericht der Deutschen Mathematiker-Vereinigung 112 (2010), 73-98.

[9] V. Klee, Diameters of polyhedral graphs, Canadian Journal of Mathematics 16 (1964), 602-614.

[10] V. Klee, Paths on polyhedra: I, Journal of the Society for Industrial and Applied Mathematics 13 (1965), 946-956.

[11] V. Klee, Paths on polyhedra: II, Pacific Journal of Mathematics 17 (1966), 249-262.

[12] V. Klee and P. Kleinschmidt, The $d$-step conjecture and its relatives, Mathematics of Operations Research 12 (1987), 718-755.

[13] B. Matschke, F. Santos, and C. Weibel, The width of 5-dimensional prismatoids, preprint, 2012, arXiv:1202.4701.

[14] F. Santos, A counterexample to the Hirsch conjecture, Annals of Mathematics 176 (2012), 383-412.

[15] F. Santos, Recent progress on the combinatorial diameter of polytopes and simplicial complexes, Top 21 (2013), 426-460.

[16] M.J. Todd, Abstract complementary pivot theory, Ph.D. thesis, Yale University, 1972.

[17] M.J. Todd, A generalized complementary pivoting algorithm, Mathematical Programming 6 (1974), 243-263.

[18] G. Ziegler, Lectures on Polytopes, Springer-Verlag, Berlin, 1995.

[19] G. Ziegler, Who solved the Hirsch conjecture? Documenta Mathematica Extra Volume: Optimization Stories (2012), 75-85. 